\title{
Ecological - hygienic soil assessment of the federal highway roadside areas
}

\author{
Anton Martsev ${ }^{1, *}$, and Oleg Selivanov ${ }^{1}$ \\ ${ }^{1}$ Vladimir State University named after A.G. and N.G. Stoletovs, 600000, 87, st. M. Gorky, Vladimir, \\ Russia
}

\begin{abstract}
Over a half (about $54 \%$ ) of all types of anthropogenic pollution belongs to transport. Soils at roadside areas experience active pollution with heavy metals, which are the most hazardous contaminants penetrating into the soil with the exhaust gases due to the increased traffic intensity of vehicles. Hygienic assessment of heavy metals content in the roadside area soils of the Federal highway "R-22" with a total length of $798 \mathrm{~km}$ has been carried out in this research. The results based on soil sampling at 11 points along the entire length of the highway at the distance of 20 and 50 $\mathrm{m}$ from the roadbed have been assessed. Soil sampling was done in compliance with the State Standard of GOST 17.4.4.02-2017 by the envelope method from the soil horizon at the depth of $0-20 \mathrm{~cm}$. HM content in soil samples was determined by the X-ray fluorescence method applying spectrometer "Spectroscan MAX-G" in compliance with the Environmental State Standards PND F 16.1.42-04. The greatest HM content was detected in the roadside area located on the black soils of spodzol, typical, southern, dark chestnut and chestnut saline soils. It testifies of the considerable soil contamination by the passing vehicles and of a real ecological harm to public health and environment. In order to reveal harmful contaminants for the population living at the roadside areas, it is recommended to organize sanitary and hygienic monitoring of soil, surface and groundwater of the roadside areas which are subject to constant HM exposure and accumulation exceeding maximum permissible concentrations (MPC) and approximate permissible amounts (APA) set by sanitary and hygienic standards.
\end{abstract}

\section{Introduction}

Over a half (about $54 \%$ ) of all types of anthropogenic pollution in Russia belongs to transport amounting about 14 million tons per year, according to the Ministry of nature report. Some sources even indicate up to 22 million tons per year [1]. The increased road

\footnotetext{
${ }^{*}$ Corresponding author: martsevaa@yandex.ru
} 
traffic intensity causes active soil contamination of the roadside areas (and in some cases they are agricultural lands) with heavy metals (HM), which are the most hazardous pollutants, coming from exhaust gases [2-4]. The ecological harm of high HM concentration is attributed by their toxic impact on soil organisms and plants. The interaction of high HM concentrations with the soil components initiates biochemical processes oppression in soil, decrease of soil microorganisms, reduction of ecosystems productivity and their ability for self-purification. Moreover HM are characterized with low mobility and high storage capacity, leading to their accumulation and long-term preservation in soil [5].

The research objective is soil ecological and hygienic assessment at the roadside areas of the highway ranking Federal importance.

\section{Materials and research methods}

The highway, connecting the cities of regional importance Ryazan and Volgograd, is a part of the Federal highway "R-22", with the length of $798 \mathrm{~km}$. The highway passes through a number of regional centers, as well as through a large number of middle and small settlements directly adjacent to the road. The research object was chosen regarding its heavy traffic along the route, especially in summer-autumn period, due to the logistics of fruit and vegetables from the southern regions of the country to the center.

The research was conducted in May 2019. In order to assess the impact of road transport on soil pollution, 11 points along the road at a distance of 20 and 50 meters from it, outside settlements and industrial enterprises (Fig. 1) were selected as the reference sites. The soil was sampled in compliance with the State Standards GOST 17.4.4.02-2017 by the envelope method from the soil horizon at a depth of $0-20 \mathrm{~cm}$.

HM content in soil samples was determined by X-ray fluorescence method applying spectrometer "SPECTROSCAN MAX-G" in accordance with Environmental State Standards PND F 16.1.42-04. Water extracts $\mathrm{pH}$ of soil samples was determined by potentiometric method using $\mathrm{pH}$ meter "Expert-001". To assess soil HM pollution level hazard coefficient $\left(K_{o}\right)$ was used, which was calculated by the formula:

$$
K_{\mathrm{o}}=\frac{C_{i}}{M P C}
$$

with: $K_{o}$ - hazard coefficient; $C_{i}-$ heavy metals content in soil $(\mathrm{mg} / \mathrm{kg})$; MPC maximum permissible concentrations of heavy metals in soil $(\mathrm{mg} / \mathrm{kg})$.

Data was processed in the program "STATISTICA". Results with a significance level of $\mathrm{p}<0.05$ were considered statistically significant. 

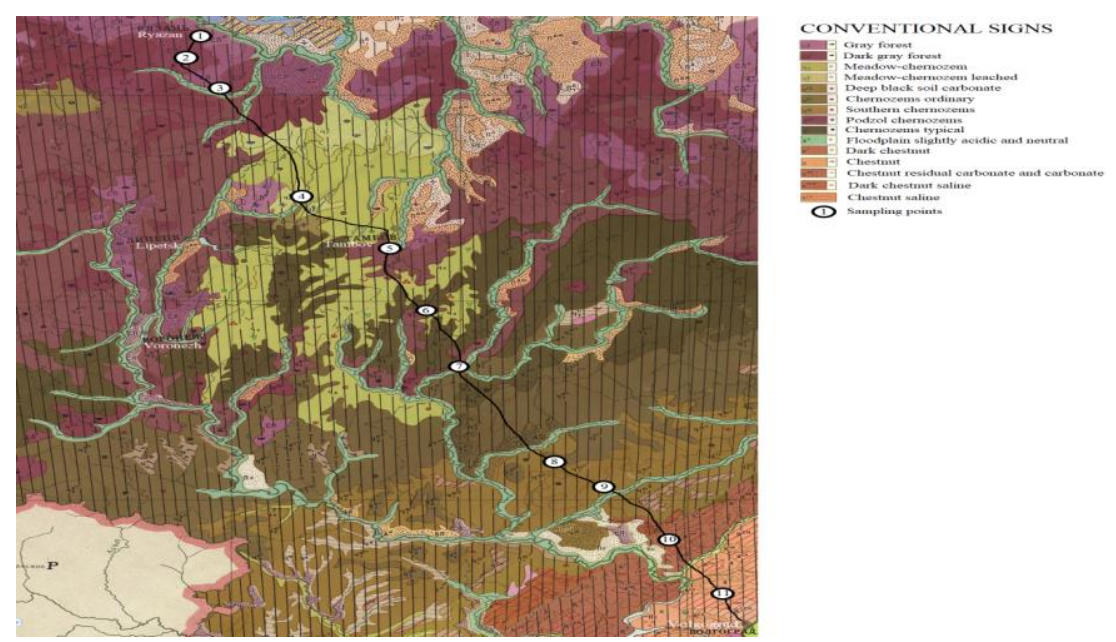

Fig. 1. Reference sampling sites with of soil type description.

\section{Results}

Soil medium $\mathrm{pH}$ plays an extremely important role in the pollutants sorption by soil compounds. It is $\mathrm{pH}$ that determines HM mobility in soils, their accumulation or leaching from it. Therefore, firstly we determined $\mathrm{pH}$ in water extracts of soil samples. The results are shown in figure 2. Water extracts $\mathrm{pH}$ of soil samples varies in the range of $5.4-7.4$, which is explained by the variety of soil types of reference sites.

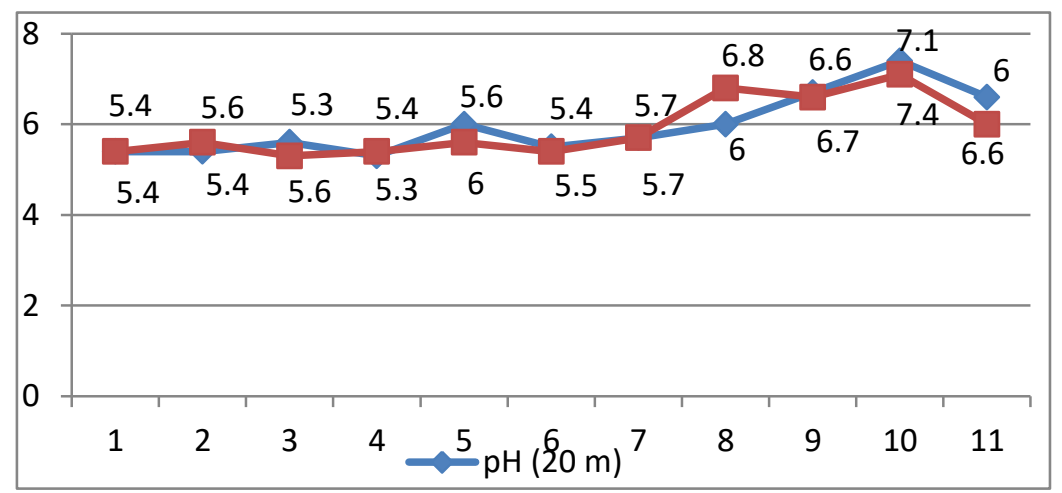

Fig. 2. Water extracts $\mathrm{pH}$ of the studied soil samples.

Soil cover zones of gray forest soils (selection points 1-3) have slightly acidic environment ( $\mathrm{pH}$ 5.1-5.5), Chernozem zone soils (sampling points 4-9) are differentiated from slightly acidic to neutral ( $\mathrm{pH} 5.4$ - 6.8), dark chestnut and chestnut (sampling points 10-11) have a neutral and slightly alkaline ( $\mathrm{pH} 6.0$ - 7.4) environment.

The soils of the roadside area are contaminated with such heavy metals as $\mathrm{Sr}, \mathrm{Pb}, \mathrm{As}$, $\mathrm{Zn}, \mathrm{Cu}, \mathrm{Ni}, \mathrm{Co}, \mathrm{Cr}, \mathrm{V}$ (table. 1). 
Table 1. Heavy metals content in soil samples

\begin{tabular}{|c|c|c|c|c|c|c|c|c|c|c|}
\hline \multirow{2}{*}{$\begin{array}{c}\text { Studied } \\
\text { soil } \\
\text { samples }\end{array}$} & \multirow{2}{*}{$\begin{array}{l}\begin{array}{c}\text { Distance } \\
\text { from } \\
\text { road, } \mathbf{m} .\end{array} \\
\end{array}$} & \multicolumn{9}{|c|}{ Heavy metals content, $\mathrm{mg} / \mathrm{kg}$} \\
\hline & & $\mathrm{Sr}$ & $\mathbf{P b}$ & As & $\mathbf{Z n}$ & $\mathbf{C u}$ & $\mathbf{N i}$ & Co & $\mathrm{Cr}$ & $\mathbf{V}$ \\
\hline \multirow[b]{2}{*}{1} & 20 & $\begin{array}{c}130,6 \\
\pm 3,6\end{array}$ & $\begin{array}{l}34,7 \\
\pm 2,5\end{array}$ & $\begin{array}{c}4,9 \\
\pm 1,0\end{array}$ & $\begin{array}{l}75,7 \\
\pm 4,1\end{array}$ & $\begin{array}{l}93,9 \\
\pm 4,3\end{array}$ & $\begin{array}{l}50,5 \\
\pm 3,2\end{array}$ & $\begin{array}{r}19,5 \\
\pm 2,7\end{array}$ & $\begin{array}{r}79,6 \\
\pm 5,1\end{array}$ & $\begin{array}{l}79,1 \\
\pm 4,3 \\
\end{array}$ \\
\hline & 50 & $\begin{array}{c}127,9 \\
+32\end{array}$ & $\begin{array}{l}31,2 \\
+24\end{array}$ & $\begin{array}{c}2,4 \\
+0,5\end{array}$ & $\begin{array}{l}70,8 \\
+38\end{array}$ & $\begin{array}{l}78,4 \\
+37\end{array}$ & $\begin{array}{l}43,9 \\
+31\end{array}$ & $\begin{array}{r}15,3 \\
+24\end{array}$ & $\begin{array}{l}76,3 \\
+48\end{array}$ & $\begin{array}{l}84,6 \\
+4.6\end{array}$ \\
\hline \multirow[b]{2}{*}{2} & 20 & $\begin{array}{c}133,1 \\
\pm 2,8\end{array}$ & $\begin{array}{r}19,8 \\
\pm 3.1\end{array}$ & $\begin{array}{c}3,3 \\
\pm 1.1\end{array}$ & $\begin{array}{l}86,3 \\
\pm 4.5\end{array}$ & $\begin{array}{r}96,1 \\
\pm 6,2\end{array}$ & $\begin{array}{r}54,0 \\
\pm 3,8\end{array}$ & $\begin{array}{r}21,5 \\
\pm 2,7\end{array}$ & $\begin{array}{r}88,9 \\
\pm 4.9\end{array}$ & $\begin{array}{r}75,7 \\
\pm 3,9\end{array}$ \\
\hline & 50 & $\begin{array}{c}125,5 \\
\pm 2,4\end{array}$ & $\begin{array}{l}13,2 \\
\pm 2,5\end{array}$ & $\begin{array}{c}1,2 \\
\pm 0,3\end{array}$ & $\begin{array}{l}74,8 \\
\pm 3,8\end{array}$ & $\begin{array}{l}70,5 \\
\pm 4.7\end{array}$ & $\begin{array}{r}49,8 \\
\pm 3.5\end{array}$ & $\begin{array}{l}17,2 \\
\pm 2.6\end{array}$ & $\begin{array}{l}85,3 \\
\pm 4.6\end{array}$ & $\begin{array}{r}75,1 \\
+36\end{array}$ \\
\hline \multirow{2}{*}{3} & 20 & $\begin{array}{l}106,1 \\
\pm 4,2\end{array}$ & $\begin{array}{r}28,2 \\
\pm 3,5\end{array}$ & $\begin{array}{c}4,2 \\
\pm 1,2\end{array}$ & $\begin{array}{r}52,9 \\
\pm 3,7\end{array}$ & $\begin{array}{l}59,3 \\
\pm 4,1\end{array}$ & $\begin{array}{l}43,8 \\
\pm 4,3\end{array}$ & $\begin{array}{l}21,6 \\
\pm 2,1\end{array}$ & $\begin{array}{l}90,1 \\
\pm 5,3\end{array}$ & $\begin{array}{r}88,9 \\
\pm 4,7\end{array}$ \\
\hline & 50 & $\begin{array}{c}101,1 \\
\pm 3,9\end{array}$ & $\begin{array}{l}12,6 \\
\pm 2,1\end{array}$ & $\begin{array}{c}3,3 \\
\pm 1,1\end{array}$ & $\begin{array}{l}46,1 \\
\pm 3,5\end{array}$ & $\begin{array}{l}58,7 \\
\pm 3,9\end{array}$ & $\begin{array}{l}37,5 \\
\pm 4,1\end{array}$ & $\begin{array}{l}14,3 \\
\pm 1,8\end{array}$ & $\begin{array}{l}78,7 \\
\pm 4,9\end{array}$ & $\begin{array}{l}84,9 \\
\pm 4,4\end{array}$ \\
\hline \multirow{3}{*}{4} & 20 & 126,8 & 18,8 & 7,5 & 93,7 & 73,5 & 60,9 & 22,4 & 116,9 & 97,1 \\
\hline & 20 & $\pm 7,1$ & $\pm 2,1$ & $\pm 1,8$ & $\pm 4,7$ & $\pm 5,1$ & $\pm 3,9$ & $\pm 2,2$ & $\pm 6,7$ & $\pm 3,5$ \\
\hline & 50 & $\begin{array}{c}116,5 \\
\pm 5,4\end{array}$ & $\begin{array}{c}3,1 \\
\pm 13\end{array}$ & $\begin{array}{c}3,3 \\
\pm 0,3\end{array}$ & $\begin{array}{l}90,1 \\
\pm 4,1\end{array}$ & $\begin{array}{l}62,9 \\
\pm 4,8\end{array}$ & $\begin{array}{l}55,2 \\
\pm 3,2\end{array}$ & $\begin{array}{l}16,6 \\
\pm 2,1\end{array}$ & $\begin{array}{l}96,7 \\
\pm 5,7\end{array}$ & $\begin{array}{l}92,9 \\
\pm 3,3\end{array}$ \\
\hline \multirow{3}{*}{5} & 20 & $\begin{array}{c}153,9 \\
\pm 5,8\end{array}$ & $\begin{array}{l}26,3 \\
\pm 3,3\end{array}$ & $\begin{array}{c}7,5 \\
\pm 2.1\end{array}$ & $\begin{array}{r}76,7 \\
+4.2\end{array}$ & $\begin{array}{c}78,8 \\
\pm 5.3\end{array}$ & $\begin{array}{l}58,1 \\
+33\end{array}$ & $\begin{array}{l}20,3 \\
\pm 27\end{array}$ & $\begin{array}{c}101,7 \\
\pm 6.1\end{array}$ & $\begin{array}{l}97,7 \\
+4.8\end{array}$ \\
\hline & & 130,9 & 17,2 & 5,1 & 69,1 & 56,6 & 50,7 & 14,4 & 95,6 & 90,9 \\
\hline & 50 & $\pm 4,1$ & $\pm 3,1$ & $\pm 1,3$ & $\pm 3,6$ & $\pm 3,2$ & $\pm 3,1$ & \pm 1.5 & \pm 5.7 & $\pm 3,5$ \\
\hline \multirow{3}{*}{6} & 20 & 142,1 & 25,4 & 1,9 & 126,9 & $\begin{array}{r}83.4 \\
+5.1\end{array}$ & 67,7 & 28,4 & 113,5 & 95,6 \\
\hline & & & $\pm 3,2$ & $\begin{array}{c} \pm 0,3 \\
130\end{array}$ & & $\frac{ \pm 5,1}{561}$ & $\frac{ \pm 4.8}{562}$ & $\pm 2,5$ & $\frac{ \pm 4,9}{1016}$ & $\frac{ \pm 2,2}{880}$ \\
\hline & 50 & $\begin{array}{c}142,3 \\
\pm 6.5\end{array}$ & $\begin{array}{l}2,1 \\
\pm 3,1\end{array}$ & $\begin{array}{l}1,30 \\
\pm 0,2\end{array}$ & $\begin{array}{l}8,4 \\
\pm 5,6\end{array}$ & $\begin{array}{r}J 0,1 \\
\pm 4,7\end{array}$ & $\begin{array}{r}0,2 \\
\pm 4,3\end{array}$ & $\begin{array}{l}18,6 \\
\pm 2,1\end{array}$ & $\begin{array}{c}101,6 \\
\pm 4,1\end{array}$ & $\begin{array}{r}88,9 \\
\pm 4,4\end{array}$ \\
\hline \multirow{3}{*}{7} & & 154,5 & 19,9 & 6,3 & 145,9 & 99,4 & 77,4 & 43,2 & 114,5 & 121,8 \\
\hline & 20 & $\pm 8,4$ & $\pm 2,5$ & $\pm 1,4$ & $\pm 6,5$ & $\pm 5,5$ & \pm 3.3 & $\pm 3,1$ & $\pm 5,8$ & $\pm 6,1$ \\
\hline & 50 & 156,8 & 13,5 & 2,5 & 86,2 & $\begin{array}{r}78,6 \\
+51\end{array}$ & 63,7 & 26,9 & 109,4 & 116,8 \\
\hline \multirow{4}{*}{8} & & 183,2 & 58,7 & 11,9 & 162,8 & 87,8 & 62,2 & 27,1 & 127,8 & 113,6 \\
\hline & 20 & $\pm 9,1$ & $\pm 4,3$ & $\pm 2,1$ & $\pm 6,7$ & $\pm 4,5$ & \pm 4.2 & $\pm 4,1$ & $\pm 6,6$ & $\pm 5,9$ \\
\hline & 50 & 165,9 & 38,4 & 9,1 & 111,5 & 87,2 & 58,2 & 28,8 & 109,3 & 103,7 \\
\hline & 50 & $\pm 8,3$ & $\pm 3,8$ & $\pm 2,0$ & $\pm 5,9$ & \pm 4.6 & \pm 3.9 & $\pm 2,5$ & \pm 5.6 & $\pm 5,7$ \\
\hline \multirow{3}{*}{9} & 20 & 227,8 & $\begin{array}{r}33,9 \\
+45\end{array}$ & $\begin{array}{r}7,5 \\
+2.1\end{array}$ & $\begin{array}{c}107,4 \\
+56\end{array}$ & $\begin{array}{l}74,1 \\
+43\end{array}$ & $\begin{array}{r}59,1 \\
+46\end{array}$ & $\begin{array}{r}33,5 \\
+29\end{array}$ & $\begin{array}{r}120,9 \\
+67\end{array}$ & 110,9 \\
\hline & 50 & 234,7 & 18,5 & 3,4 & 89,9 & 70,7 & 58,9 & 25,3 & 102,2 & 99,9 \\
\hline & 50 & $\pm 11,2$ & \pm 2.5 & $\pm 0,8$ & $\pm 5,0$ & $\pm 4,2$ & \pm 4.1 & \pm 2.1 & \pm 5.4 & $\pm 6,8$ \\
\hline \multirow{4}{*}{10} & 20 & 129,9 & 52,2 & 8,7 & 132,5 & 73,9 & 46,8 & 13,9 & 112,3 & $\begin{array}{r}75,7 \\
\end{array}$ \\
\hline & 20 & $\pm 7,3$ & $\pm 3,5$ & $\pm 1,8$ & $\pm 6,4$ & $\pm 4,4$ & $\pm 3,7$ & $\pm 1,6$ & $\pm 6,2$ & $\pm 3,8$ \\
\hline & & 122,5 & 40,9 & 4,5 & 65,5 & 57,1 & 45,8 & 10,9 & 98,8 & 68,5 \\
\hline & 50 & \pm 6.8 & $\pm 3,2$ & $\pm 0,5$ & $\pm 2,9$ & $\pm 3,7$ & $\pm 3,6$ & $\pm 1,4$ & $\pm 4,8$ & \pm 3.6 \\
\hline \multirow{4}{*}{11} & 20 & 256,9 & 47,1 & 7,5 & 87,9 & 78,9 & 59,6 & 30,4 & 117,4 & 90,9 \\
\hline & 20 & $\pm 10,3$ & $\pm 3,6$ & $\pm 1,3$ & $\pm 6,7$ & $\pm 6,4$ & $\pm 4,5$ & $\pm 3,9$ & $\pm 5,8$ & $\pm 6,1$ \\
\hline & 50 & 172,2 & 15,9 & 5,4 & 71,2 & 67,3 & 61,5 & 27,1 & 115,7 & 87,1 \\
\hline & 50 & $\pm 8,5$ & $\pm 2,1$ & $\pm 1,2$ & $\pm 5,8$ & $\pm 4,6$ & $\pm 5,1$ & $\pm 3,2$ & $\pm 4,9$ & $\pm 5,4$ \\
\hline \multirow{2}{*}{$\begin{array}{c}\text { Average } \\
\text { value }\end{array}$} & 20 & 154,5 & 33,2 & 5,5 & 107,7 & 81,7 & 58,1 & 23,9 & 107,8 & 95,2 \\
\hline & 50 & 149,2 & 20,4 & 3,7 & 77,6 & 66,2 & 52,8 & 19,6 & 97,2 & 84,1 \\
\hline MPC & & $*$ & 32 & 2 & 100 & 55 & 85 & $25^{* * *}$ & $100^{* * * * 4}$ & 150 \\
\hline
\end{tabular}

* - MPC for strontium in soils is not normalized;

** - MPC for the gross form of cobalt is taken from the Guidelines [6];

*** MPC for $\mathrm{Cr}$ (III)

To assess the level of soil cover pollution, hazard coefficients $\left(\mathrm{K}_{\mathrm{o}}\right)$ are calculated for each of them (table. 2). 
Table 2. Hazard coefficients $\left(\mathrm{K}_{\mathrm{o}}\right)$ of heavy metals in soil samples.

\begin{tabular}{|c|c|c|c|c|c|c|c|c|c|c|}
\hline \multirow[b]{2}{*}{$\begin{array}{c}\text { Studied } \\
\text { soil } \\
\text { samples }\end{array}$} & \multirow[b]{2}{*}{$\begin{array}{l}\text { Distance } \\
\text { from } \\
\text { road, } m .\end{array}$} & \multicolumn{9}{|c|}{ Hazard coefficient, $K_{0}$} \\
\hline & & $\mathbf{P b}$ & As & $\mathbf{Z n}$ & $\mathrm{Cu}$ & $\mathbf{N i}$ & Co & $\mathrm{Cr}$ & V & $\begin{array}{c}\Sigma \\
\text { average } \\
\text { value }\end{array}$ \\
\hline \multirow{2}{*}{1} & 20 & 1,08 & 2,45 & 0,75 & 1,70 & 0,59 & 0,39 & 0,79 & 0,52 & 1,03 \\
\hline & 50 & 0,97 & 1,20 & 0,71 & 1,42 & 0,51 & 0,30 & 0,76 & 0,56 & 0,80 \\
\hline \multirow{2}{*}{2} & 20 & 0,61 & 1,65 & 0,86 & 1,74 & 0,63 & 0,43 & 0,89 & 0,50 & 0,91 \\
\hline & 50 & 0,41 & 0,6 & 0,75 & 1,28 & 0,58 & 0,34 & 0,85 & 0,50 & 0,66 \\
\hline \multirow{2}{*}{3} & 20 & 0,88 & 2,1 & 0,53 & 1,08 & 0,51 & 0,43 & 0,90 & 0,59 & 0,88 \\
\hline & 50 & 0,39 & 1,65 & 0,46 & 1,06 & 0,44 & 0,28 & 0,79 & 0,56 & 0,70 \\
\hline \multirow[b]{2}{*}{4} & 20 & 0,58 & 3,75 & 0,94 & 1,33 & 0,71 & 0,44 & 1,17 & 0,64 & 1,20 \\
\hline & 50 & 0,09 & 1,65 & 0,90 & 1,14 & 0,65 & 0,33 & 0,96 & 0,61 & 0,79 \\
\hline \multirow{2}{*}{5} & 20 & 0,82 & 3,75 & 0,76 & 1,43 & 0,68 & 0,40 & 0,99 & 0,65 & 1,19 \\
\hline & 50 & 0,53 & 2,55 & 0,69 & 0,73 & 0,59 & 0,28 & 0,95 & 0,60 & 0,87 \\
\hline \multirow{2}{*}{6} & 20 & 0,79 & 0,95 & 1,26 & 1,55 & 0,79 & 0,56 & 1,13 & 0,63 & 0,96 \\
\hline & 50 & 0,72 & 0,65 & 0,88 & 1,02 & 0,66 & 0,37 & 1,01 & 0,59 & 0,74 \\
\hline \multirow{2}{*}{7} & 20 & 0,62 & 3,15 & 1,46 & 1,80 & 0,91 & 0,86 & 1,14 & 0,81 & 1,34 \\
\hline & 50 & 0,41 & 1,25 & 0,86 & 1,43 & 0,74 & 0,47 & 1,09 & 0,77 & 0,88 \\
\hline \multirow{2}{*}{8} & 20 & 1,82 & 5,95 & 1,62 & 1,59 & 0,73 & 0,54 & 1,27 & 0,75 & 1,78 \\
\hline & 50 & 1,20 & 4,55 & 1,01 & 1,58 & 0,68 & 0,57 & 1,09 & 0,69 & 1,42 \\
\hline \multirow{2}{*}{9} & 20 & 1,05 & 1,75 & 0,97 & 1,34 & 0,69 & 0,67 & 1,21 & 0,73 & 1,05 \\
\hline & 50 & 0,57 & 1,70 & 0,90 & 1,28 & 0,69 & 0,50 & 1,02 & 0,66 & 0,92 \\
\hline \multirow{2}{*}{10} & 20 & 1,63 & 4,35 & 1,32 & 1,34 & 0,55 & 0,27 & 1,12 & 0,55 & 1,39 \\
\hline & 50 & 1,27 & 2,25 & 0,65 & 1,03 & 0,53 & 0,21 & 0,99 & 0,45 & 0,92 \\
\hline \multirow{2}{*}{11} & 20 & 1,47 & 3,75 & 0,87 & 1,43 & 0,70 & 0,60 & 1,17 & 0,60 & 1,32 \\
\hline & 50 & 0,49 & 2,7 & 0,71 & 1,22 & 0,72 & 0,54 & 1,15 & 0,58 & 1,01 \\
\hline \multirow{2}{*}{\multicolumn{2}{|c|}{ Average value $K_{o}$}} & 1,03 & 2,75 & 1,06 & 1,48 & 0,68 & 0,47 & 1,07 & 0,63 & 1,15 \\
\hline & & 0,64 & 1,85 & 0,77 & 1,20 & 0,62 & 0,39 & 0,97 & 0,56 & 0,86 \\
\hline
\end{tabular}

It has been revealed that along the highway on average, $K_{o}$ indicators for lead exceed MPC by 1.03 times, for arsenic-2.75 times, for zinc-1.06 times, for copper-1.48 times, for chromium-1.07 times. The average hazard coefficients for $\mathrm{Ni}$, Co and $\mathrm{V}$ are below one, i.e. roadside contamination with these pollutants is below MPC at almost all sampling sites.

The maximum values of $K_{o}$ for single pollutants are revealed in southern chernozems for $\mathrm{Pb}-1,82$, As -5,95, for $\mathrm{Zn}-1,62$, for $\mathrm{Cr}-1,27$, in chernozems typical for $\mathrm{Cu}-1,8$.

\section{Conclusions}

1. It is established that as an emissions result of the passing vehicles, roadside pollution excessing MPC values with such $\mathrm{HM}$ as $\mathrm{Pb}, \mathrm{Zn}, \mathrm{Cu}, \mathrm{Cr}$ and metalloid As reliably occurs.

2. $\mathrm{Pb}, \mathrm{As}, \mathrm{Zn}, \mathrm{Cr}, \mathrm{Cu}$ of southern and typical chernozems are characterized by biggest hazard coefficients.

3. To identify hazardous pollutants threatening the population health the living in the roadside area, it is necessary to organize sanitary and hygienic monitoring of soil, surface and groundwater of the roadside areas subject to constant exposure and accumulation of HM exceeding MPC and approximate permissible amounts (APA) set by sanitary and hygienic standards.

4. Maximum limitations for using old vehicles, transition to the gas fuel and to the gasoline "Euro-5", the increase of environment friendly transport such as electric and hybrid vehicles are considered to be the most important factors for reducing the damage of roadside areas.

5 . 


\section{References}

1. T.A. Trifonova, A.A. Podolec, O.G. Selivanov, A.A. Martsev, A.A. Podolec, Theoret. App. Ecolo., №2, 94-101 (2018)

2. T.A. Trifonova, A.A. Martsev, Hyg. Sanit., 94 (4),14-18 (2015)

3. YA.O. Timofeeva, New. Irk. Stat.Univ. Seri. Biol. Eco., T.6.№3, 94-99 (2013)

4. M. R. Hafen, R. Brinkmann, Enviro. Geoc. Heal., 18(4), 171-179 (2001)

5. M. Werkenthin, B. Kluge, G. Wessolek, Environ. Pollu., 189, 98-110 (2014)

6. O. A. Neverova, I. N. Egorova, Wor. App. Sci. J., 23(5), 650-655 (2013) 